\title{
HISTORIA
}

\section{HISTORIA DE LA ESCUELA DE ENFERMERAS «SALUS INFIRMORUM» DE MADRID}

\author{
Miralles Sangro, $\mathrm{M}^{\mathrm{a}} \mathrm{T}$. \\ Enfermera Hospital Ramón y Cajál. Profesora Asc. EUE y F Universidad de Alcalá \\ Garre Murúa, E. \\ Subdirectora EUE y F Salus Infirmorum, Madrid. Univ.Pontificia de Salamanca \\ CASAs MarTínez, $\mathrm{M}^{\mathrm{a}} \mathrm{F}$. \\ Profesora Titular Interina de la EUE y F Universidad de Alcalá \\ RUIZ UREÑA, T. \\ Profesora Titular de la EUE y F. Universidad de Alcalá \\ González Villanueva, P. \\ Enfermera Hospital Ramón y Cajál. Profesora Asc. EUE y F Universidad de Alcalá
}

\author{
Correspondencia: $\mathrm{M}^{\mathrm{a}}$ Teresa Miralles Sangro \\ Samaria ${ }^{\circ} 4,3^{\circ}$ izq. $\bullet 28009$ Madrid \\ Teléfono - $91 / 4.09 .38 .98$
}

\section{RESUMEN}

El objetivo del estudio es describir el origen de la Escuela de Enfermeras «Salus Infirmorum» de Madrid, inaugurada en el año 1943 con unas características específicas, que desde nuestro punto de vista se han mantenido fieles a su origen, y conocer su evolución hasta nuestros días. Para ello comenzamos con una visión retrospectiva de la asistencia socio- sanitaria en el Madrid de la postguerra y de los Organismos e Instituciones encargados de la formación de las enfermeras en la época.

Las fuentes utilizadas fueron: Revisión bibliográfica. Normativas, Estatutos, publicaciones periódicas y documentos originales existentes en la Institución «Salus Infirmorum», así como Entrevistas in situ enfocadas y biográficas. El desarrollo del trabajo recoge las etapas mas significativas en la evolución de la Escuela: La Institución Salus Infirmorum, su fundadora. Inauguración de la Escuela. Ampliación de Estudios. Escuela de Enfermeras-ATS. Escuela Universitaria de Enfermería. Los resultados encontrados constatan como mas significativo:

- La Escuela de Enfermeras Salus Infirmorum nace para cubrir las necesidades de formación en un momento determinado y lejos de extinguirse en el tiempo, es capaz de adaptarse a la realidad social de nuestros días.

- Desde su fundación, La Escuela de Salus Infirmorum ha mantenido la finalidad para la que fue creada: Preparar al estudiante desde las vertientes ; científica, técnica, humana, ética y cristiana.

Palabras clave: Historia de la Enfermería. Salus Infirmorum. Escuela Enfermeras. Formación de Enfermeras

\section{HISTORY OF THE MADRID NURSING SCHOOL «SALUS INFIRMORUM»}

\section{ABSTRACT}

The aim of the present study is first: to describe the origin of the Nursing School «Salus Infirmorum», opened in Madrid in 1943, with its specific characteristics. According to our personal opinion the School has been faithful to its origin. Second: to discover its evolution from the beginning up to the present time. 
We started by giving a retrospective view to the socio-sanitary care in the Madrid after-war, as well as to regarding the Institutions and Social Organizations in charge of the nursing education at that time.

Sources used were: Bibliographical revisions. Rules, Legislations, Laws, periodical publications and original documents available in the «Salus Infirmorum»School, as well as personal interviews built up for the purpose and biographical interviews.

The study shows the most significant stages and evolution of the Nursing School: The Institution Salus Infirmorum. The lady founder. The Opening of the School. Further Study Programme. Nursing-ATS School. Nursing School attached to University.

The results show as the most significant facts:

- The Nursing School of «Salus Infirmorum» appears to cover the education needs of a very special moment and far from extinguishing later on, the School adapts itself to the social reality of the present time.

- Fron its foundation, the School of «Salus Infirmorum» has been faithful to the aims of its conception: To furnish the student with scientific, technical, human, ethic and christian education and criteria.

Key words: Nursing History. Salus Infirmorum. Nursing School. Nursing Education.

\section{INTRODUCCION}

El trabajo que presentamos pretende contribuir al conocimiento de nuestra historia profesional, reconociendo las causas que contribuyeron a que distintas iniciativas y filosofías establecieran centros para la formación de enfermeras. En los años posteriores a la guerra civil española, en Madrid, la población sufre pobreza y falta de recursos sanitarios. La iglesia y grupos seglares afines siguen manteniendo un gran número de centros de asistencia, de distinto nivel, para enfermos y necesitados.

Son característicos de esta época los Dispensarios Parroquiales donde se proporcionaba asistencia sanitaria ambulatoria en dos situaciones diferentes. Por un lado se asistía a los necesitados en el mismo Dispensario ; pasando la consulta con el médico, administrando medicamentos, curando heridas, tomando constantes. Por otro lado, se programaban visitas al domicilio de los enfermos que no podían acudir al Dispensario, y allí, en su propia casa, se les proporcionaban cuidados higiénicos a los pacientes encamados, se llevaba la medicación desde el Dispensario y se administraban los medicamentos prescritos por el médico, se hacían curas y las actividades concretas prescritas por el médico (Ilustración 1). La formación de las enfermeras en este periodo de posguerra estuvo marcada por la variedad de centros e instituciones, tanto públicas como privadas, donde se formaban profesionalmente : enfermeras militares, matronas, practicante, enfermeras visitadoras, enfermeras de la Cruz Roja, enfermeras del Cuerpo de la Falange Española Tradicionalista y de las JONS. (Santo Tomás Pérez, 1994). El 21 de mayo de 1941, una Orden Ministerial establece que los estudios para obtener el título de enfermera deben tener una duración de dos años y ajustarse a los planes de estudio aprobados por las Facultades de Medicina, o en centros reconocidos por ellas, como por ejemplo la Cruz Roja. Se exige escolaridad y una edad mínima de 18 años y máxima de 45. (Hernández Martín, 1996)

\section{MATERIAL Y MÉTODOS}

Para el estudio se revisaron y recogieron datos de diversas Normativas, Estatutos, publicaciones periódicas y documentos originales existentes en la Institución Salus Infirmorum. También se realizaron entrevistas, seleccionando como informantes a personas de la Institución Salus Infirmorum que han sido testigos y / o protagonistas de la situación estudiada. El estudio es descriptivo y se refiere a las etapas mas significativas en la evolución de la Escuela de Enfermeras.

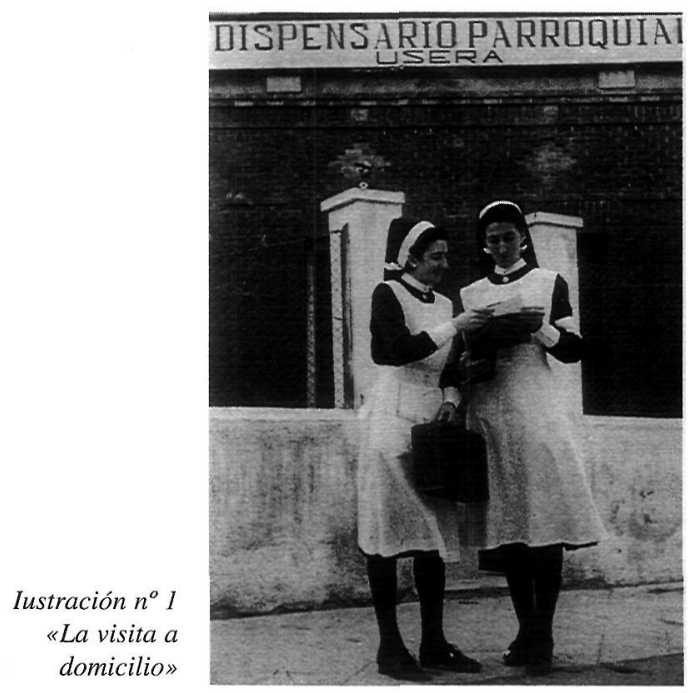




\section{DESARROLLO}

\section{SALUS INFIRMORUM. SU FUNDADORA}

María de Madariaga Alonso nace en Madrid, el año 1906. Mujer profundamente católica, idealista, tenaz y constante, comienza su trabajo de apostolado a los 18 años, en la Unión de Damas Apostólicas. Posteriormente, se incorpora a la Acción Católica, desde donde colabora directamente con la Jerarquía de la Iglesia, en servicios sociales, caritativos y de formación de las jóvenes. Desde estos momentos tuvo el propósito de organizar grupos, dentro de la Acción Católica, por profesiones y sobretodo empezar por la enfermería

Es nombrada Presidenta Diocesana y Nacional de la Juventud Femenina de Acción Católica en 1926. Su contacto con los enfermos y su dedicación a los cuidados le llevan a realizar un curso de formación enfermera en el Hospital del Niño Jesús de Madrid, en el año 1936, posteriormente realiza los estudios de enfermera en la Facultad de Medicina de Madrid. Movida por el sentido de beneficencia en dispensarios y hospitales, y conocedora de la labor de las enfermeras, cree necesario crear un centro especializado, donde atender y actualizar los conocimientos de las enfermeras integradas en la Acción Católica.

El día 18 de julio de 1936 se fija para la apertura del Centro pero el proyecto no llegó a realizarse, pues empezó la guerra civil. En 1941, es distinguida con la Cruz «Pro ecclesia et Pontifice», otorgada por Su Santidad Pio XII.

En el año 1942, María de Madariaga que no ha desistido en su empeño de atender y mejorar la formación de las enfermeras, funda con la ayuda de la Iglesia, la ASOCIACIÓN CATÓLICA DE HERMANDADES DIOCESANAS DE SANITARIAS ESPAÑOLAS, dán-
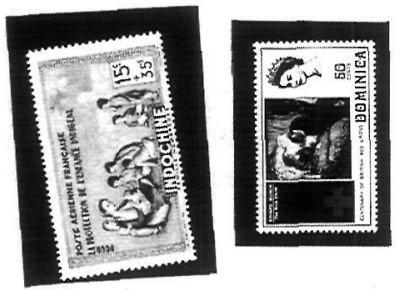

dole a esta Asociación el nombre de Salus Infirmorum, advocación de la Virgen en la letanía. El 26 de Enero de 1944 Salus Infirmorum queda erigida canónicamente por el Patriarca Obispo
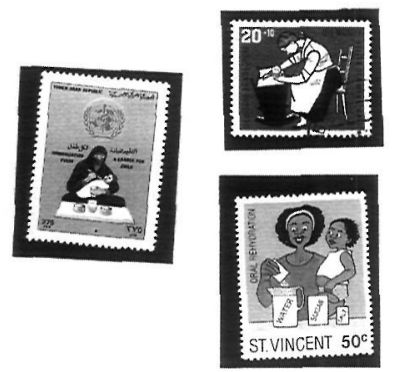
de Madrid-Alcalá, Dr. D. Leopoldo Eijo y Garay, en la Iglesia de las Comendadoras de Santiago, en la Diócesis de Madrid. En el 1946, a los dos años de su funda- ción, fue declarada Hermandad Nacional por S.E. el Cardenal Primado de Toledo, Dr.D. Enrique Pla y Daniel, quién en 1947 ratificó la Documentación, Junta y Nombramientos Nacionales; y así quedó constituida la ASOCIACIÓN CATÓLICA NACIONAL «SALUS INFIRMORUM», donde quedan definitivamente agrupadas confesionalmente todas las sanitarias españolas. La ASOCIACIÓN se incorpora, en el año 1947, al Comité Internacional Católico de Enfermeras y Asistencia Médica y Social, CICIAMS, como Miembro Adherido, (máxima categoría dentro de este organismo). El 25 de junio de 1953, la Entidad Salus Infirmorum fue registrada para legalizar su existencia y actividad, con éste nombre, en la Dirección General de Seguridad-Jefatura Superior de Policía, con el $\mathrm{n}^{\circ}$ de Referencia de Asociaciones: 8.256. Posteriormente la Dirección General de Política Interior ratificó su legalidad, confirmando su inscripción con el número nacional 4.680, del Registro Nacional de Asociaciones.

En 1960 se agrega a la Asociación el personal auxiliar, surgido de las necesidades sanitarias del país, que ha incorporado al Equipo Asistencial de los Servicios Sanitarios éste otro nivel. Su finalidad ha sido promover una acción apostólica, capaz de llevar a cabo, a través de sus miembros, la evangelización al mundo de la salud, por medio del testimonio cristiano, su capacitación profesional y la adhesión incondicional al Sumo Pontífice. (Ilustración 2) Salus Infirmorum, creada por María de Madariaga, ha abierto ocho Escuelas de Enfermeras (ATS) oficialmente reconocidas por el Estado y por la Jerarquía de la Iglesia: Madrid, Cuenca, La Coruña, Cádiz, Salamanca, Palma de Mallorca, Tánger y Valladolid. Una Escuela de Diplomadas de niños en Madrid, Regina Angelorum. Una Escuela de Fisioterapéutas en Madrid. Una Escuela de Matronas en Cádiz. Una Residencia y un Club para enfermeras en Madrid.

En 1946 se le concede la medalla de plata otorgada por la Cruz Roja Española

En 1947 representa a España en la primera reunión del Comité Internacional Católico de Enfermeras y Asistencia Medico Social, (CICIAMS), celebrado en Roma.

En 1950 fue elegida Vicepresidenta Internacional del CICIAMS María de Madariaga crea y dirige durante toda su publicación (1952-1981) el Boletín de Sanitarias Católicas de España. Responsable de la Comisión Permanente de Moral Profesional del CICIAMS, cargo designado en la reunión celebrada en Bruselas el año 1958 En 1963 recibe la Officier de l'Ouissan Alaouit distinción del reino de Marruecos.

Por O.M. del 2 de abril de 1973, ingresa en la Orden Civil de Beneficencia, con distintivo blanco y categoría 
de Cruz de $1^{a}$ Clase, $D^{a}$ María de Madariaga Alonso, Presidenta de Salus Infirmorum. Ostenta el cargo de Presidenta General de Salus Infirmorum de forma vitalicia, por deseo expreso de sus miembros y ratificado por la Jerarquía de la Iglesia.

\section{INAUGURACIÓN DE LA ESCUELA}

Para el logro de sus fines, Salus Infirmorum inicia en 1943 la preparación de un futuro sanitario con el propósito de actualizar, revalorizar y especializar a todas las profesionales del campo de la enfermería, a través de la Obra de la Iglesia. Se inaugura la Escuela de Enfermeras SALUS INFIRMORUM con el curso 1942/43, vinculándose al Hospital del Niño Jesús de Madrid.

El Plan de Estudio se abrió a cinco vertientes: científico, técnico, humano, ético y espiritual. El programa de estudios para este primer curso contemplaba las asignaturas de religión, ética profesional, moral, nociones de anatomía y fisiología, higiene, física y química, cultura general y cocina dietética. Además de las clases teóricas se contemplaba la asistencia a las Prácticas, asistiendo primero durante dos meses a la Sala de Demostración y posteriormente a las Prácticas Clínicas en las salas del Hospital. La preparación Básica Enfermera se realizará mediante dos años de carrera y un curso más de Especialidad para las alumnas que quisieran culminar sus

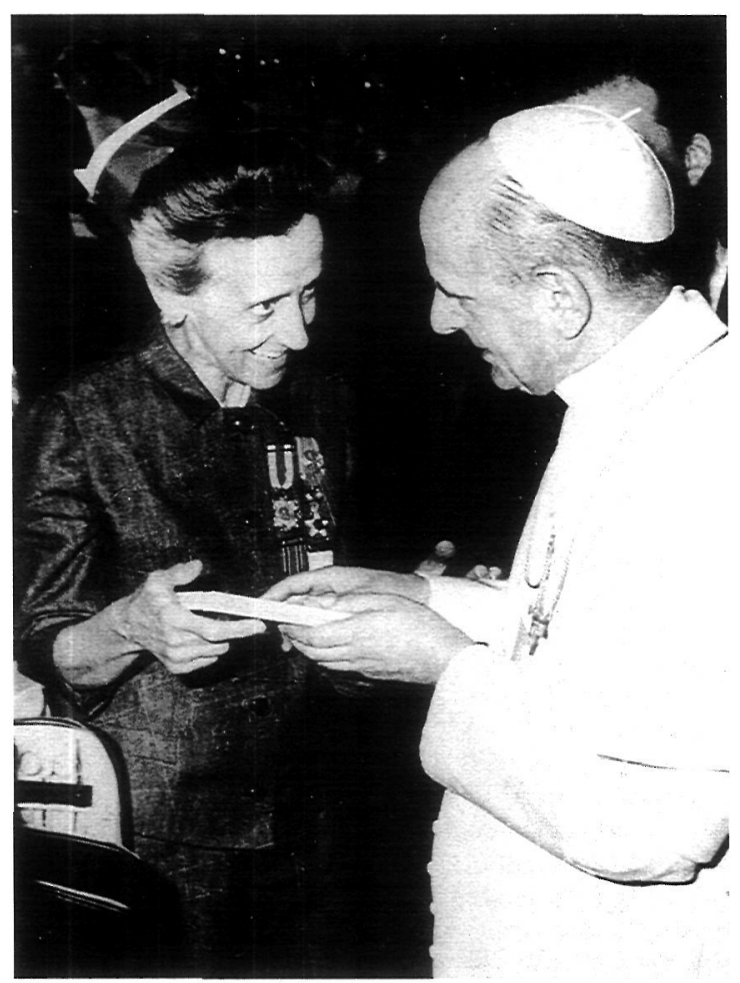

Ilustración 2 estudios en las ramas de : psiquiatría, cirugía, puericultura y pediatría, radiología o laboratorio.

Simultáneamente, las alumnas que no hubieran cursado estudios de bachillerato, debían seguir durante los dos años de la carrera, enseñanzas de cultura general para así completar su formación.

Ostentaba la Dirección de la Escuela el Profesor D.Gregorio Marañón Posadillo, y la Jefatura de la Escuela la Sra. Da María Benavente. El profesorado estaba formado por médicos del Hospital del Niño Jesús, para las clases teóricas, y las Enfermeras Tituladas impartieron clases prácticas en la Sala de Demostración y tutelaron las prácticas clínicas del Hospital.

El uniforme de las alumnas de la Escuela lo diseñó la Hermana Mayor (María de Madariaga) : Vestido gris, largo y amplio. Delantal de tela blanca fuerte, con peto, en forma de capa, casi cerrado detrás y con bolsillo en el lado derecho. Cuello y cinturón duros y con brillo. Toca de tela blanca almidonado muy duro. Medias negras. Zapatos negros abotinados, con tacón bajo o medio. Guantes para diario azul marino, para gala, de hilo blanco. Abrigo azul marino, suelto, de hechura raglán. Cinturón negro. Insignia con anagrama de la Escuela.

Alumnado quince aspirantes a enfermeras terminaron sus estudios en este primer curso de la Escuela.

\section{AMPLIACIÓN DE ESTUDIOS. TRES AÑOS DE FORMACIÓN BÁSICA}

En el curso 1947/48 se amplían los estudios de la carrera de enfermera hasta tres años de formación básica, cursados año por año única forma de obtener el diploma, que otorgaba la Escuela y que era independiente del Título Oficial que expedía la Facultad de Medicina de la Universidad Complutense.

Por el contrario, el Título Oficial se lograba después de dos años de estudios, mediante la superación de un examen ante un Tribunal de la Facultad de Medicina, y la presentación de un Certificado de Prácticas expedido por un médico. (Dos cursos que podían efectuarse en una misma convocatoria, sin necesidad de escolarización). El Plan de Estudios desde este curso hasta el año 1953 incluía: Lengua Castellana, Religión, Matemáticas, Química, Biología, Anatomía, P. General, P. Quirúrgica, Pediatría, Puericultura, Obstetricia y Ginecología, Higiene General, Dietética, Enfermería Fundamental, Asepsia, Oxigenoterapia, Formación Familiar y Social, Moral, Ética Profesional, y Formación Espiritual.

En cuanto a las prácticas, se introdujo una nueva modalidad, además de las hospitalarias, se amplió esta formación al área extrahospitalaria. Las alumnas de Tercer Curso, acompañadas por Enfermeras Domiciliarias de la Asociación, una vez por semana prestaban asistencia en 
los Dispensarios Parroquiales de los suburbios del cinturón de Madrid. De esta forma se abrió el campo de la COMUNIDAD a la Enfermería. (Los planes de estudio gubernamentales lo incluyen por primera vez en los Planes de Estudio de Enfermería Universitaria). La Dirección de la Escuela estuvo a cargo del Profesor Marañón. El cargo de Rectora de la Escuela lo ejerció $D^{a}$ $\mathrm{M}^{\mathrm{a}}$ Rosa Cardenal Turul, enfermera miembro de Salus Infirmorum. Profesores médicos impartieron las clases teóricas (50 horas) y dos profesoras licenciadas las clases de Cultura General (50 horas). Se pautaron exámenes trimestrales eliminatorios, presididos por la Hermana Mayor y Profesorado de la Escuela, no presentando a examen en la Facultad de Medicina de San Carlos más que a las alumnas que los hubieran superado.

Las Prácticas Hospitalarias fueron tutorizadas por Enfermeras Tituladas de Salus Infirmorum y se realizaron en el Hospital de la Princesa, el Hospital Provincial, el Hospital de Niño Jesús, el Sanatorio de la Consolación. Se redactó el «Reglamento de la Escuela de Enfermeras de Salus Infirmorum», en el que se hace referencia a la asistencia, la puntualidad, la disciplina, la uniformidad y la aplicación. En el periodo 1947/52 finalizaron sus estudios de Enfermera 173 alumnas.

\section{ESCUELA DE ENFERMERAS - ATS}

A comienzos del año 1952 Salus Infirmorum es requerida por el Ministerio de Educación y Ciencia para formar parte, como Vocal, de la Comisión Central de los Estudios de Enfermera. Salus Infirmorum aportó su experiencia de diez años.

Por Decreto de 27 de junio de 1952 se modifican los estudios de Enfermería y en su lugar se establecen las Escuelas de Ayudantes Técnicos Sanitarios. Al amparo de esta ley y ajustándose a la legislación promulgada, Salus Infirmorum crea y organiza en 1953 la Escuela de Ayudantes Técnicos Sanitarios en la Diócesis de Madrid, obteniendo el reconocimiento oficial como Escuela de la Iglesia, el 24 de abril de 1954, y adscrita a la Facultad de Medicina de la Universidad de su distrito. Conservando plena «autonomía» tanto en el terreno económico como ideológico.

El 17 de febrero de 1954 fue aprobada definitivamente al amparo de la O.M. de 4 de agosto de 1953 que dictó las normas para la aprobación oficial de las Escuelas de fundación NO OFICIAL.

Vinculación de la Escuela al Hospital Clínico de San Carlos.- Durante la década 1955 a 1965, la Escuela de ATS queda vinculada a efectos de prácticas y docencia al Hospital Clínico de San Carlos, dependiente de la Facultad de Medicina de la Universidad Complutense, hasta que diez años mas tarde la propia facultad crea la suya. Todas las asignaturas correspondientes al área de ciencias medicas se imparten en las Aulas de la Cátedra del Dr.Marañón, que seguía siendo el director de la Escuela hasta su fallecimiento.

Plan de Estudios 1953-1966.-. Total horas Teóricas 945, horas Prácticas 2.500

Formación del personal docente de la Escuela.- Para formar a las futuras Instructoras de la nueva Escuela de ATS, Salus Infirmorum envió a sus Enfermeras a Europa. Unas a la Universidad de Lovaina para formarse como docentes, otras a centros hospitalarios de Francia, Italia, Bélgica e Inglaterra y mas tarde a Irlanda para completar su formación, ampliar sus estudios y conocer nuevas técnicas.

Clausura del Hospital Clínico de San Carlos y Vinculación a la Ciudad Sanitaria de la SS «La Paz».-Al trasladarse la Facultad de Medicina de San Carlos al actual Hospital Clínico en el años 1965 y crear éste su propia Escuela de ATS, el Ministerio de Educación y Ciencia de común acuerdo con el de Trabajo, acuerdan vincular la Escuela de ATS Salus Infirmorum, a efectos de docencia y prácticas, a la Ciudad Sanitaria de la Seguridad Social «La Paz».

Ostentaba la Dirección de la Escuela el Dr. D. Francisco Cano Iglesias, que después del fallecimiento del del Dr. Marañón, le sucedió en su cargo. Rectora de la Escuela en este periodo fué $\mathrm{D}^{\mathrm{a}}$ Mercedes Porras-IslaFernandez.

Estructura y evolución del plan de estudios.- Ante la necesidad urgente de actualizar el Plan de Estudios Oficial de la carrera de ATS, que desde su publicación en 1955, no había sufrido modificación alguna, se hace un reajuste del programa de cada área, se añaden nuevas asignaturas y otras se modifican. El Plan de Estudios de los años 1966/1980 comprendía 1.208 horas teóricas y 2.300 horas prácticas

Formación integral. Actividades extraescolares.- La Escuela de Salus Infirmorum ha tenido siempre como

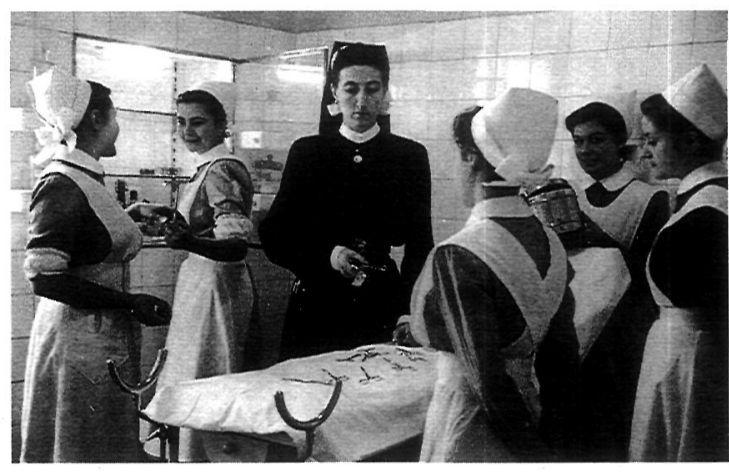

Ilustración 3. Sala de demostraciones 
objetivo LA FORMACIÓN INTEGRAL del alumno por lo que desde sus comienzos se incluyendo en el Plan de Estudios una serie de actividades extraescolares, tanto en el ámbito nacional como en el internacional. Se organizaron también Foros Culturales, sistemáticos, en las aulas de la Escuela.

Número de alumnos. - Un total de 922 alumnas fueron tituladas como Enfermeras de Salus Infirmorum en el periodo que transcurre entre los años 1953 al 1980

\section{ESCUELA UNIVERSITARIA DE ENFERMERÍA SALUS INFIRMORUM}

El 23 de julio de 1977 se aprueba el R.D: 2128/1977 que recoge la integración de las Escuelas de ATS en la Universidad como Escuelas Universitarias de Enfermería, con lo que desaparece definitivamente la figura de los Ayudantes Técnicos Sanitarios (ATS). La Escuela Universitaria de Enfermería, Salus Infirmorum, obtiene su reconocimiento oficial, a efectos civiles por parte del Ministerio de Educación y Ciencia por Real Decreto 1579 en el año 1982 y es un Centro Adscrito a la Universidad Pontificia de Salamanca desde 1980. (Hernández Conesa, 1995)

Objetivos de la Escuela:

- Encuadra su actividad docente dentro del derecho y del deber de la función evangelizadora de la Iglesia Católica, animada de espíritu cristiano y de respeto a la libertad y dignidad humana.

- Reclama la visión «integral del hombre», no fraccionado sino individualizado e irrepetible, para servirle en su totalidad.

- Apoya, desde la Fé y la razón, el estudio y la investigación para una docencia cualificada y el correcto ejercicio profesional de Enfermeras, por su naturaleza universitaria

- Presenta con gozo la búsqueda del bien y la verdad, para que sus alumnos intenten descubrirla y comunicarla en el amplio campo de la salud y de la sociedad.

- Fomenta el amor al «saber».

- Atiende y defiende toda la trayectoria de la vida humana desde la concepción; los problemas: del desarrollo del hombre; de la salud y la enfermedad o incapacidad; de la sociedad y de propia Iglesia, a la luz de la Ética Cristiana. (Documento Original)
El Plan de Estudios vigente, aprobado según Resolución de 20 de marzo de 1997, de la Dirección General de Enseñanza Superior contempla un total de carga lectiva de 3.920 horas, distribuidas en 1.315 horas teóricas y 2.605 horas de prácticas clínicas.

En la actualidad el Director de la Escuela es el Dr. D. Lorenzo Rubio Morales, Jefe de Estudios Dr. Nistal Martín de Serrano, Subdirectora Técnica $D^{a}$ Elena Garre Murua, Secretaria $D^{a}$ Mercedes Porras-Isla-Fernandez. Forman el cuadro de Profesores, Médicos y Enfermeras. Asisten a los alumnos en sus prácticas clínicas Monitoras Enfermeras.

\section{CONCLUSIONES}

Constatan como más significativos los siguientes aspectos:

- La Escuela de Enfermeras Salus Infirmorum nace para cubrir las necesidades de formación en un momento determinado y lejos de extinguirse en el tiempo, es capaz de adaptarse a la realidad social de hoy.

- Los Planes de Estudio se han estructurado conforme a los requerimientos sociales y académicos en las diferentes etapas.

- Las Prácticas Clínicas que realizan los alumnos se han enfocado de acuerdo a los conocimientos teóricos impartidos con el propósito de proporcionar al alumno la posibilidad de saber «que hacer», «porqué» y «para qué».

- Desde su fundación, la Escuela de Salus Infirmorum ha mantenido la finalidad para la que fue creada: Preparar al estudiante desde las vertientes; científica, técnica, humana, ética y cristiana.

\section{BIBLIOGRAFÍA}

Santo Tomás Pérez, M. (1994) Enfermería Fundamental «Historia de la Enfermería». Masson, Barcelona.

Hernádez Martín, F. (1996). Historia de la Enfermería. Síntesis, Madrid .

Hernández Conesa, J. (1995).Historia de la Enfermería. McGraw-Hill-Interamericana, Madrid

Normativas, Estatutos, Publicaciones periódicas y documentos originales existentes en la Institución «Salus Infirmorum». 